\title{
Bloodstream infections in patients with hematological malignancies: which is more fatal - cancer or resistant pathogens?
}

This article was published in the following Dove Press journal:

Therapeutics and Clinical Risk Management

17 September 2014

Number of times this article has been viewed

\author{
Habip Gedik' \\ Funda Șimșek' \\ Arzu Kantürk' \\ Taner Yildirmak' \\ Deniz Arica ${ }^{2}$ \\ Demet Aydin ${ }^{2}$ \\ Naciye Demirel ${ }^{2}$ \\ Osman Yokuș² \\ 'Department of Infectious \\ Diseases and Clinical Microbiology, \\ ${ }^{2}$ Department of Hematology, Ministry \\ of Health Okmeydanı Training and \\ Research Hospital, İstanbul, Turkey
}

Correspondence: Habip Gedik İnfeksiyon Hastalıkları ve Klinik Mikrobiyoloji Kliniği, Bakırköy Sadi Konuk Eğitim ve Araștırma Hastanesi,

İstanbul, Türkiye

Tel +902 2। 23145555

Fax +90 21 2221 7800

Email habipgedik@yahoo.com
Background: The primary objective of this study was to report the incidence of bloodstream infections (BSIs) and clinically or microbiologically proven bacterial or fungal BSIs during neutropenic episodes in patients with hematological malignancies.

Methods: In this retrospective observational study, all patients in the hematology department older than 14 years who developed febrile neutropenia during chemotherapy for hematological cancers were evaluated. Patients were included if they had experienced at least one neutropenic episode between November 2010 and November 2012 due to chemotherapy in the hematology ward.

Results: During 282 febrile episodes in 126 patients, 66 (23\%) episodes of bacteremia and 24 (8\%) episodes of fungemia were recorded in 48 (38\%) and 18 (14\%) patients, respectively. Gram-negative bacteria caused 74\% (n=49) of all bacteremic episodes. Carbapenem-resistant Gram-negative bacteria $(n=6)$ caused $12 \%$ and $9 \%$ of Gram-negative bacteremia episodes and all bacteremia episodes, respectively. Carbapenem-resistant Gram-negative bacteria included Acinetobacter baumannii $(\mathrm{n}=4)$, Pseudomonas aeruginosa $(\mathrm{n}=1)$, and Serratia marcescens $(\mathrm{n}=1)$. Culture-proven invasive fungal infection occurred in 24 episodes in 18 cases during the study period, with 15 episodes in ten cases occurring in the first study year and nine episodes in eight cases in the second study year. In 13 of 18 cases (72\%) with bloodstream yeast infections, previous azole exposure was recorded. Candida parapsilosis, C. glabrata, and C. albicans isolates were resistant to voriconazole and fluconazole.

Conclusion: BSIs that occur during febrile neutropenic episodes in hematology patients due to Gram-negative bacteria should be treated initially with non-carbapenem-based antipseudomonal therapy taking into consideration antimicrobial stewardship. Non-azole antifungal drugs, including caspofungin and liposomal amphotericin B, should be preferred as empirical antifungal therapy in the events of possible or probable invasive fungal infections with an absence of pulmonary findings due to increase azole resistance.

Keywords: bloodstream infection, fungemia, bacteremia, carbapenem resistance, azole resistance, antifungal treatment, hematological malignancies, febrile neutropenia

\section{Introduction}

Infections are common complications in hematology patients. Patients with hematological malignancies are at increased risk of infections, not only because of the malignancy itself, but also because of neutropenia induced by intensive chemotherapy and its cytotoxic effect on the cells that line the gastrointestinal tract. ${ }^{1}$ The survival rate is related to the prevention and management of infectious complications. Febrile neutropenia is a complication of chemotherapy and should be taken into consideration in assessment of treatment choice. Infection risk of hematological patients with neutropenia 
has been assessed according to Infectious Diseases Society of America guidelines as being described high risk (prolonged neutropenia, $>7$ days; neutrophil count $\leq 100 / \mathrm{mm}^{3}$; substantial concurrent comorbidity; clinically unstable) and low risk (neutropenia expected to resolve within 7 days). Whereas Gram-negative bacteria (GNB; eg, Escherichia coli, Klebsiella spp., and Pseudomonas aeruginosa) predominated in neutropenic cancer patients in the 1970s and early 1980s, Gram-positive bacteria (coagulase-negative staphylococci and viridans streptococci) gradually started to predominate in the late 1980s and early 1990s. ${ }^{2,3}$ In a case-control study by Pagano et al, risk factors for bacteremia in patients with hematological malignancies were found to be use of a central venous catheter and neutropenia for more than 6 days. ${ }^{4}$ Candidemia is still a serious disease in hematology patients, although the incidence of candidemia has reduced with the introduction of azole drugs in the 1990s..$^{5}$ The candidemias generally develop endogenously through invasion of the gastrointestinal tract or as a catheter-related infection. ${ }^{6,7}$ It is recommended that an empirical antifungal drug be considered in patients at high risk who have persistent fever over 4-7 days of broad-spectrum antibacterial therapy and no identified source of fever. ${ }^{8}$

The primary objective of this study was to report the incidence of bloodstream infections (BSIs) and clinically or microbiologically proven bacterial or fungal BSIs during neutropenic episodes in patients with hematological malignancies.

\section{Materials and methods}

In this retrospective observational study, all patients older than 14 years who developed febrile neutropenia during chemotherapy for hematological cancers in the hematology department between November 2010 and November 2012 were evaluated. The local ethics committee of Ministry of Health Okmeydanı Training and Research Hospital approved this study. Patients were included if they had experienced at least one neutropenic episode due to chemotherapy in the hematology ward. Patients treated for other hematological diseases (eg, anemia, idiopathic or immune thrombocytopenia purpura) or had not been screened by rectal swab culture for vancomycin-resistant enterococci (VRE) colonization while in the hematology ward were excluded.

The hematology department is equipped with 23 beds in single, double, and quadruple rooms without high-efficiency particulate air filters at the Ministry of Health Okmeydanı Training and Research Hospital, an 800-bed tertiary hospital in İstanbul, Turkey. In the hematology ward, patients and their attendants reside in the same room and share three toilets.

A weekly 1-hour instructional program regarding drug-resistant microorganisms and preventative measures was presented to patients and their attendants by a nurse and a doctor in the hematology ward. Febrile neutropenia was defined as an oral temperature $>38.3^{\circ} \mathrm{C}$ or two consecutive readings $>38.0^{\circ} \mathrm{C}$ for 2 hours and an absolute neutrophil count $<0.5 \times 10^{9} / \mathrm{L}$ or a count expected to fall below $0.5 \times 10^{9} / \mathrm{L}^{8}{ }^{8}$ The collected data included patient demographics and diagnosis, neutropenic episodes, clinical presentations and laboratory findings, antimicrobial treatments, microbiological data, and outcomes. In our hospital, management of febrile neutropenia is based on the clinical practice guidelines of the Infectious Diseases Society of America, the European Organization for Research and Treatment of Cancer/Invasive Fungal Infections Cooperative Group, and the National Institute of Allergy and Infectious Diseases Mycoses Study Group. ${ }^{1,7,8}$ The scoring system for risk of complications in febrile neutropenic patients was based on Multinational Association for Supportive Care in Cancer (MASCC) score. ${ }^{8}$

Blood samples drawn from a vein or catheter were inoculated into BactAlert ${ }^{\circledR}$ 3D bottles (bioMérieux Diagnostics, Lyon, France). In addition, samples of urine, sputum, wound, conjunctiva, abscess, blood, and catheter were inoculated onto 5\% sheep blood agar (Salubris Inc., Istanbul, Turkey), or chocolate agar (Salubris Inc.) and MacConkey agar (Salubris Inc.). Isolated yeasts from blood cultures were identified by morphological examination on Sabouraud dextrose agar plates (Unipath Ltd., Basingstoke, UK), examination of germ tube formation, and API ID 32C (bioMérieux Diagnostics) for discrimination at the species level. An ATB Fungus 2 microdilution kit (bioMérieux Diagnostics) was used for susceptibility testing according to the Clinical and Laboratory Standards Institute (formerly National Committee for Clinical Laboratory Standards) broth microdilution M27-A2 protocol. An Aspergillus galactomannan antigen test was performed using a commercial enzyme immunoassay kit (Platelia ${ }^{\mathrm{TM}}$ Aspergillus immunoenzymatic sandwich microplate assay; Bio-Rad Laboratories, Marnes-la-Coquette, France). Blood samples from patients were analyzed twice weekly and the results were computed as an index whereby values $\geq 0.5$ were considered as positive relative to an optical density of the control sample measured with a semiautomatic analyzer (enzyme-linked immunosorbent assay processor III; Dade Behring, Marburg, Germany). ${ }^{9}$ Possible causes of false-positive and negative results were excluded. The test 
was considered significant if the patient had two consecutive Aspergillus galactomannan antigen tests with an index of $\geq 0.5$ under persistent or recurrent fever after 4-7 days of antibiotics with or without microbiological or radiological findings associated with fungal infection, as mentioned earlier, and the overall duration of neutropenia was expected to be more than 7 days. ${ }^{8}$ Catheters were removed if the patient did not respond to antifungal therapy, with deteriorating clinical, radiological, and laboratory findings. Catheters were not removed in patients who needed a central line to receive treatment intravenously due to trouble with the intravenous route.

Bile esculin azide agar plates containing $6 \mu \mathrm{g} / \mathrm{mL}$ of vancomycin (Becton, Dickinson and Company, Sparks, MD, USA) were used to screen the patients for VRE colonization. After inoculating rectal swabs, the plates were subsequently incubated aerobically at $35^{\circ} \mathrm{C}-37^{\circ} \mathrm{C}$ with $5 \%-10 \% \mathrm{CO}_{2}$ for up to 48 hours. Samples from the patients were cultured at 2-week intervals. VRE colonization was defined as a positive culture from a rectal swab, and colonization continued until two consecutive rectal swab cultures were negative. Patients colonized or infected with VRE or carbapenem-resistant (CR) GNB underwent cohorting. A patient sharing a room with another patient colonized or infected with CR GNB was screened with swabs rubbed in the armpit, groin, and rectal areas. Invasive procedures performed in VRE-colonized patients included placement of chemotherapy port catheter lines or central line catheters and bone marrow biopsy during follow-up. Patients and their attendants were informed about resistant microorganisms and appropriate preventive measures by a nurse and doctor for 1 hour each week while residing in the ward. Patients were encouraged to use alcohol-based hand disinfectant after contact with contaminated or potentially contaminated materials and zones. Patients colonized with VRE resided in the same room. Health care workers were advised to use gloves and a gown when entering the room if contact with patient body fluids was possible. The hospital floors were cleaned daily with sodium hypochlorite $(1,000$ parts per million). Use of glycopeptide and antianaerobic antibiotics was restricted.

The therapeutic regimens that were active against $P$. aeruginosa included piperacillin-tazobactam, cefoperazonesulbactam, and piperacillin-tazobactam in combination with ciprofloxacin, in accordance with local antibiotic resistance status. Antibacterial treatment was changed to either imipenem or meropenem or to other antibiotics effective against bacteria grown in the sample culture if the patient had a persistent fever after 2 days of empirical antibiotic therapy or had clinical, laboratory, and radiological findings. Vancomycin was administered according to the previously mentioned guidelines. Patients with VRE bacteremia were treated with linezolid $2 \times 600 \mathrm{mg}$ /day for at least 14 days. Patients with vancomycin-sensitive enterococci (VSE) were treated with ampicillin-sulbactam 8-12 g/day plus gentamicin 160-240 mg/day for at least 14 days. Patients who had bacteremia caused by CR GNB were treated with Colimycin ${ }^{\circledR}$. The recommended dose of this drug is $2.5-5 \mathrm{mg} / \mathrm{kg}$ of colistin base per day, which equals $6-12 \mathrm{mg} / \mathrm{kg}$ of colistimethate sodium per day. The drug was administered as monotherapy or in combination with another active antibiotic (eg, rifampicin, aminoglycosides, tigecycline) for at least 14 days after receiving susceptibility results. Antibiotic doses were adjusted if the patient experienced hepatic or renal failure. Antifungal and antiviral treatments were not considered in this study, as they were not relevant to the aim or content of the study.

Empirical antifungal treatment was considered for patients with persistent or recurrent fever after 4-7 days of antibiotics and whose overall duration of neutropenia was expected to be more than 7 days. If computed tomography scans showed changes associated with fungal infection, liposomal amphotericin B or voriconazole was initiated. Voriconazole was continued as oral therapy after the infection responded to systemic therapy and the patient was able to receive oral therapy. Cavitation, the air crescent sign, and the halo sign were classified as the major changes for invasive pulmonary aspergillosis (IPA). Nodules and new infiltrates, including consolidation and effusions, were classified as minor changes. ${ }^{10}$ Possible IPA was defined as highly suggestive clinical and radiologic findings of infection without histopathologic and/or microbiologic evidence of infection. Probable IPA was defined as a positive culture for Aspergillus species from a respiratory specimen or two consecutive Aspergillus galactomannan antigen tests with an index of $\geq 0.5$ and clinical and radiologic findings suggestive of IPA. Proven IPA was defined as histopathologic evidence of tissue invasion and damage by Aspergillus species with clinical and radiologic findings. ${ }^{11}$ First-line therapy was defined as the antifungal treatment given initially. Salvage therapy was defined as an antifungal therapy that was given after antifungal treatment that did not achieve clinical and microbiological responses. Posaconazole was used for primary antifungal prophylaxis and given as $200 \mathrm{mg}$ orally three times daily with food and beverage, which increases the absorption of the drug during the time that the neutrophil count is decreased to 
below $1 \times 10^{9} / \mathrm{L}$ subsequent to chemotherapy until recovered to $1 \times 10^{9} / \mathrm{L}$. Secondary antifungal prophylaxis was administered to patients who had received antifungal therapy previously for IPA as voriconazole $200 \mathrm{mg}$ twice daily orally or posaconazole $200 \mathrm{mg}$ three times daily during the time that the neutrophil count decreased to below $1 \times 10^{9} / \mathrm{L}$ subsequent to chemotherapy until recovered to $1 \times 10^{9} / \mathrm{L}$. If patient could not receive oral therapy, secondary antifungal prophylaxis was administered intravenously. Antibiotic prophylaxis was not administered to these patients.

A treatment response was defined as resolution of fever in the 48-72 hours following the initiation of antimicrobial therapy and all laboratory findings and clinical symptoms related to infection. Inhospital mortality during the neutropenic phase and the clinical outcomes of febrile neutropenic episodes were the primary outcomes investigated in this study.

\section{Statistical analysis}

Variables, including age and patient MASCC scores, are shown as the mean \pm standard deviation and range. The infection-related mortality rate was calculated as the ratio of deaths affected by infections to the total number of included patients in the study year. The fatality rate of bacteremia caused by CR GNB was calculated as the ratio of deaths affected by CR GNB bacteremia to the total number of patients with bacteremia caused by CR GNB. Mortality rates related to invasive fungal infection (IFI) were calculated as the ratio of deaths affected by IFI to the total number of included patients in the study year. Mortality rates related to candidemia were calculated as the ratio of deaths affected by candidemia to the total number of patients with candidemia in the study year and two study years. Overall 30-day crude mortality rates were calculated as the ratio of deaths within 30 days of development of neutropenia to the number of included patients in the study year.

\section{Results}

During the study period, 15 of 141 patients admitted to the hematology ward were excluded for not meeting the study criteria. In total, 282 febrile episodes in 126 consecutive patients with neutropenia were retrospectively analyzed during the study period, with 65 cases examined in the first study year and 78 in the second study year. The mean patient age was $51.73 \pm 14.4$ (range 17-82) years and 60 patients were female. The mean MASCC score was 17.18 \pm 8.27 (Table 1). The mean duration of febrile neutropenia was $29.38 \pm 6.95$ days. During 282 febrile episodes in 126 patients, 66 (23\%) episodes of
Table I Hematologic malignancies

\begin{tabular}{ll}
\hline Hematologic malignancies & $\mathbf{n}(\%)$ \\
\hline Acute myeloblastic leukemia & $73(58)$ \\
Acute lymphocytic leukemia & $22(17)$ \\
Non-Hodgkin lymphoma & $7(5)$ \\
Chronic lymphocytic leukemia & $5(4)$ \\
Multiple myeloma & $5(4)$ \\
Hairy cell leukemia & $4(3)$ \\
Aplastic anemia & $3(2)$ \\
Chronic myeloid leukemia & $2(2)$ \\
Plasma cell leukemia & $2(2)$ \\
Mantle cell lymphoma & $2(2)$ \\
Chronic lymphocytic leukemia with Burkitt's lymphoma & I (1) \\
Total & $\mathbf{I} \mathbf{2 6}(\mathbf{1 0 0})$ \\
\hline
\end{tabular}

bacteremia and 24 (8\%) episodes of fungemia were recorded in $48(38 \%)$ and $18(14 \%)$ patients, respectively.

\section{Bacterial BSIs}

GNB caused $74 \%(n=49)$ of all bacteremia episodes. CR GNB $(n=6)$ caused $12 \%$ and $9 \%$ of Gram-negative bacteremia episodes and all bacteremia episodes, respectively. Clinical and microbiological responses were achieved using either piperacillin-tazobactam or cefoperazone-sulbactam therapy in $76 \%$ (32/42) of the cases with bacteremia caused by carbapenemsensitive GNB. A treatment response was achieved by switching from non-carbapenem-based beta-lactam antipseudomonal antibiotics to carbapenem-based therapy in $17(25 \%)$ bacteremia episodes caused by extended-spectrum beta-lactamase (ESBL)-producing Klebsiella pneumoniae $(\mathrm{n}=5)$, non-ESBL-producing E. coli $(\mathrm{n}=5)$, ESBL-producing E. coli $(\mathrm{n}=2)$, non-ESBL-producing $K$. pneumoniae $(\mathrm{n}=2)$, Ochrobactrum anthropi $(\mathrm{n}=1)$, and $P$. aeruginosa $(\mathrm{n}=2)$.

CR GNB included Acinetobacter baumannii ( $\mathrm{n}=4)$, P. aeruginosa $(\mathrm{n}=1)$, and Serratia marcescens $(\mathrm{n}=1$; Table 2$)$. The fatality rate was $50 \%$ among six patients with bacteremia caused by CR GNB as a result of two deaths associated with CR A. baumannii and one case of death associated with CR $P$. aeruginosa. Colistin monotherapy was administered to two patients with CR $A$. baumannii and one patient with $\mathrm{CR}$ $S$. marcescens. One patient who received colistin monotherapy died. Colistin combined with rifampicin was administered to a patient with CR $A$. baumannii and a patient with $\mathrm{CR}$ $P$. aeruginosa. Colistin combined with sulbactam-cefoperazone was administered to a patient with CR A. baumannii, who died. The hematological cancers of those patients were acute myeloid leukemia (AML) in three cases, non-Hodgkin lymphoma in one case, multiple myeloma in one case, and acute lymphoblastic leukemia in one case. The IFIs of the cases who died of bacteremia were under antifungal treatment and regressing at the time of death. All patients with CR GNB 
Table 2 Bacteria isolated from bloodstream infections in hematology patients

\begin{tabular}{|c|c|c|}
\hline & n (\%) & Fatal isolate $(n)$ \\
\hline Extended-spectrum beta-lactamases (-) Escherichia coli & $14(2 \mid)$ & 2 \\
\hline Extended-spectrum beta-lactamases (-) Klebsiella pneumoniae & $8(12)$ & \\
\hline Vancomycin-sensitive Enterococcus faecalis & $6(9)$ & 3 \\
\hline Carbapenem-sensitive Pseudomonas aeruginosa & $6(9)$ & \\
\hline Extended-spectrum beta-lactamases $(+) K$. pneumoniae & $5(8)$ & \\
\hline Carbapenem-resistant Acinetobacter baumannii & $4(6)$ & 2 \\
\hline Methicillin-resistant Staphylococcus aureus & $4(6)$ & 2 \\
\hline Extended-spectrum beta-lactamases $(+)$ E. coli & $3(5)$ & \\
\hline Methicillin-sensitive S. aureus & $2(3)$ & \\
\hline Vancomycin-resistant Enterococcus faecium & $2(3)$ & I \\
\hline Vancomycin-sensitive E. faecium & $2(3)$ & I \\
\hline Ochrobactrum anthropi & $\mathrm{I}(\mathrm{l} .5)$ & \\
\hline Methicillin-resistant coagulase-negative staphylococci & $\mathrm{I}(\mathrm{I} .5)$ & \\
\hline Extended-spectrum beta-lactamases (-) Klebsiella oxytoca & $\mathrm{I}(\mathrm{I} .5)$ & \\
\hline Stenotrophomonas maltophilia & $\mathrm{I}(\mathrm{I} .5)$ & \\
\hline Carbapenem-resistant $P$. aeruginosa & $\mathrm{I}(\mathrm{I} .5)$ & I \\
\hline Carbapenem-resistant Serratia marcescens & $\mathrm{I}(\mathrm{I} .5)$ & \\
\hline S. marcescens & $\mathrm{I}(\mathrm{I} .5)$ & \\
\hline Serratia ficaria & $\mathrm{I}(\mathrm{I} .5)$ & \\
\hline Citrobacter koseri & $\mathrm{I}(\mathrm{I} .5)$ & \\
\hline Enterobacter cloacae & $\mathrm{I}(\mathrm{I} .5)$ & \\
\hline Total & $66(100)$ & \\
\hline
\end{tabular}

were followed up and isolated in the hematology ward. Screen cultures from rectal, axillary, and groin sites were negative in these cases. CR GNB developed in these patients during carbapenem therapy used to treat febrile neutropenic episodes. Central or port line catheters were removed from patients who had bacteremia caused by CR GNB after identification. $\mathrm{CR}$ isolates developed during carbapenem therapy that was administered to treat febrile neutropenic episodes.

Fifty (39.68\%) patients were colonized with VRE, and the mean number of VRE colonization days per patient was $34.27 \pm 13.12$ days. VRE bacteremia developed in two (4\%) patients during a total of 1,295 colonization days, including a male patient with non-Hodgkin's lymphoma who survived the infection and a female patient with AML who died from VRE bacteremia. The hematological malignancies in the patients with VSE-related bacteremia and bacteriuria were AML ( $n=3)$, acute lymphocytic leukemia $(n=1)$, multiple myeloma $(n=1)$, non-Hodgkin's lymphoma $(n=1)$, and hairy cell leukemia $(n=1)$. Two patients with VSE bacteremia died.

\section{Fungal BSls}

Over the 2-year period, 18 cases of fungemia in 24 febrile neutropenia episodes, 19 cases of probable IPA infection in 25 febrile neutropenia episodes, 38 cases of possible IPA infection in 42 febrile neutropenia episodes, 30 cases of suspected IFI in 31 episodes, and five cases (5\%) of hepatosplenic candidiasis were diagnosed and treated. Of the 24 episodes in 18 cases with fungemia, 15 episodes in ten cases occurred in the first study year and nine episodes in eight cases in the second study year. Previous azole exposure was recorded in 13 of 18 cases $(72 \%)$ with bloodstream yeast infections. Candida parapsilosis, Candida glabrata, and Candida albicans isolates were resistant to voriconazole and fluconazole (Table 3). Due to availability of posaconazole only in the second study year, patients could receive primary posaconazole prophylaxis. Only one patient with a colostomy could not tolerate because of diarrhea among 13 patients whom received primary posaconazole prophylaxis. Only seven (53\%) patients required systemic antifungal treatment under induction or consolidation chemotherapy. Systemic antifungal therapy was administered owing to possible $(\mathrm{n}=13)$ or probable $(\mathrm{n}=6)$ IFIs in 16 of $38(42 \%)$ and three of eight (37\%) febrile neutropenia episodes among 26 patients who were administered secondary antifungal prophylaxis with oral voriconazole $(n=17)$ or posaconazole $(n=6)$ over 46 episodes.

\section{Mortality}

Overall 30-day crude mortality rates were calculated as $50 \%(23 / 65)$ in cases diagnosed with AML $(n=16)$, acute lymphocytic leukemia $(n=5)$, multiple myeloma $(n=1)$, or chronic myeloid leukemia $(n=1)$ in the first study year and $21 \%(17 / 78)$ in patients diagnosed with AML $(n=16)$, acute lymphoblastic leukemia $(n=4)$, or non-Hodgkin lymphoma $(n=1)$ in the second study year. AML was the most important risk factor in patients who died. The number of patients 


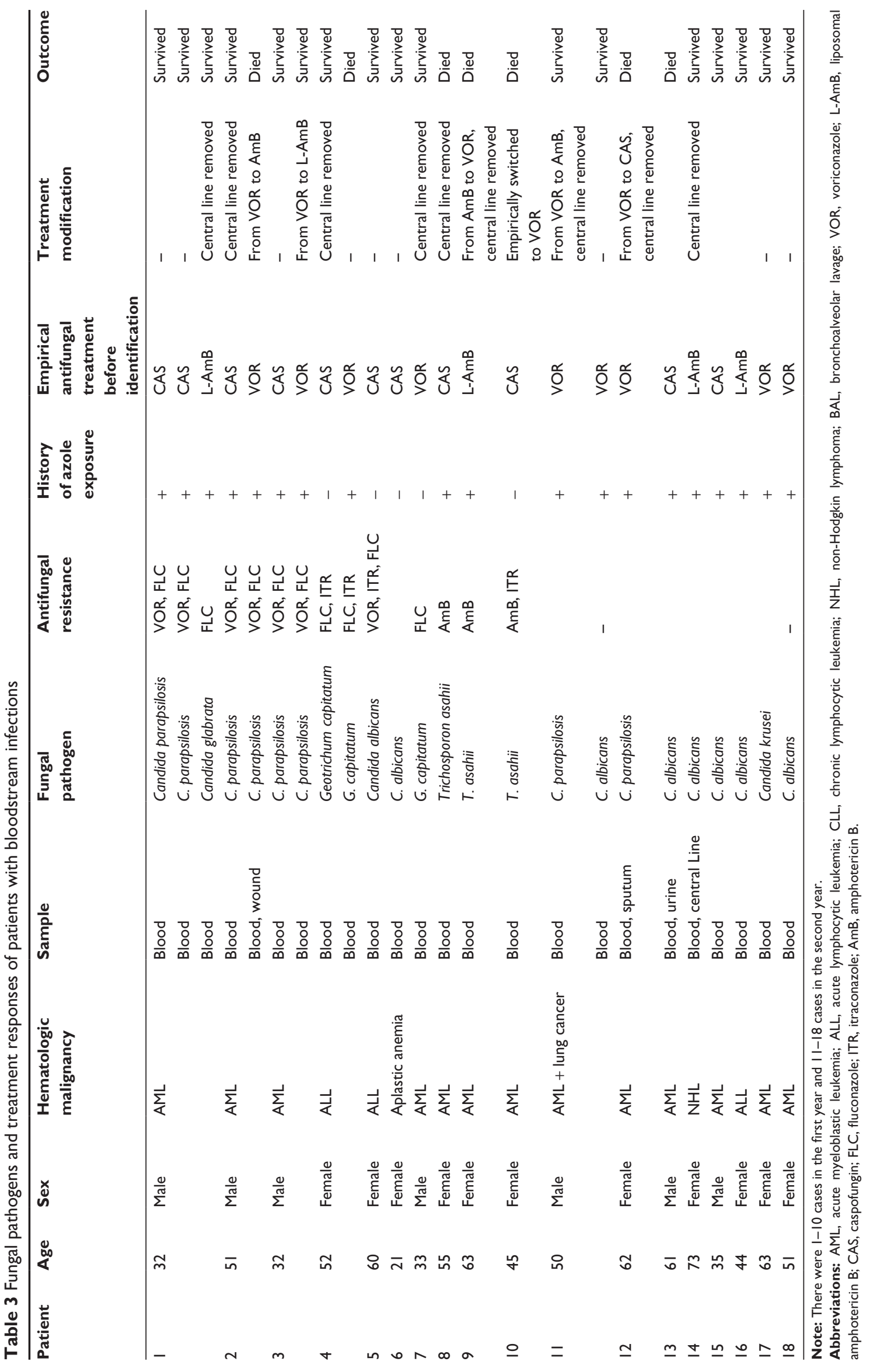


who died of infections was $17(26 \%)$ in the first study year and eleven (14\%) in the second study year. Methicillinresistant Staphylococcus aureus bacteremia (n=2), vancomycin-sensitive Enterococcus faecium-induced severe sepsis $(\mathrm{n}=1)$, bacteriuria caused by vancomycin-resistant Enterococcus faecalis $(\mathrm{n}=1)$, and fungemia caused by Trichosporon asahii $(\mathrm{n}=3)$, Candida kefyr $(\mathrm{n}=1)$, Geotrichum capitatum $(\mathrm{n}=1)$, and $C$. parapsilosis $(\mathrm{n}=1)$ were the causes of death in the first study year. Probable IFI $(n=1)$ and primary BSIs associated with $\mathrm{CR}$ A. baumannii $(\mathrm{n}=2), \mathrm{CR}$ $P$. aeruginosa $(\mathrm{n}=1)$, non-ESBL-producing $E$. coli $(\mathrm{n}=2)$, vancomycin-sensitive $E$. faecalis $(\mathrm{n}=2)$, vancomycin-resistant E. faecium $(\mathrm{n}=1)$, C. albicans $(\mathrm{n}=1)$, and C. parapsilosis $(\mathrm{n}=1)$ were the causes of death in the second study year. Mortality rates associated with IFI were $7 \%(n=5)$ in the first study year, $3 \%(n=3)$ in the second study year, and $6 \%(n=8)$ in overall (Table 2). The mortality rates related to candidemia were $33 \%$ ( $1 / 9$ episodes) in the first study year, $22 \%$ (2/9 episodes) in the second study year, and 16\% (3/18 episodes) overall.

\section{Discussion}

BSI occurred in half of the study population during the study period. Bacteria, especially GNB, were responsible for the BSI episodes in our hematology patients, similar to previous studies reporting that GNB were commonly isolated pathogens in patients hospitalized due to cancer or other diseases. ${ }^{12-15}$ Endogenous flora that contain mainly Enterobacteriaceae and other GNB in the gastrointestinal tract can cause bacteremia or candidemia episodes in patients with neutropenia due to breakdown of mucosal barriers secondary to chemotherapy. ${ }^{16}$ Of the bacteremia episodes caused by GNB, Enterobacteriaceae comprised the majority of isolates in accordance with breakdown of mucosal barriers secondary to chemotherapy. Whilst CR has been a big problem worldwide currently, carbapenem-sensitive and non-ESBL producing GNB predominantly caused bacteremia in the hematological patients. ${ }^{17}$ Age, duration of neutropenia, presence of an indwelling central venous catheter, hematological malignancy, previous use of cefepime, and use of total parenteral nutrition have been described as risk factors for BSI by multidrug-resistant GNB. ${ }^{18-20}$ Prolonged neutropenia and use of multiple broad-spectrum antibiotics due to febrile neutropenic episodes admit of selection and colonization of resistant bacteria in the gut and other body sites of patients. Those colonized bacteria cause bacteremia episodes passing through the damaged mucosal barriers due to chemotherapy as occurred in our patients. Mortality rates associated with CR GNB have been reported to be between
40\% and 65\%. ${ }^{21,22}$ Acute Physiology and Chronic Health Evaluation (APACHE) score $>20$, septic shock, and inappropriate antimicrobial therapy are independent risk factors associated with mortality due to BSI caused by multidrug resistant GNB. ${ }^{19-21}$ Chemotherapy port catheter lines in patients who had CR GNB were removed in accordance with the guideline. ${ }^{8}$ Infection control measures and antimicrobial stewardship are the basic measures used to control these multidrug-resistant microorganisms. Due to the fact that frequent and prolonged use of carbapenems and other broader-spectrum antibiotics have been increasing antibiotic resistance worldwide, non-carbapenem antipseudomonal antibiotics, including piperacillin-tazobactam and cefoperazone-sulbactam, which achieved clinical and microbiological responses in three quarters of the cases, should be chosen for febrile neutropenic episodes in hematology patients. Only one quarter of the cases needed carbapenem therapy.

Persistent fever, which is not a reason to escalate antimicrobial therapy to carbapenems, often prompts physicians to switch to carbapenem-based therapy in hematology patients with the idea that delayed broader-spectrum antibiotic therapy can cause fatal consequences. ${ }^{8,15}$ Infection control measures, including cohorting, environmental decontamination, contact precautions, surveillance, and screening of the patients who shared the room with other colonized or infected patients with CR-GNB or VRE are likely to have reduced the infection associated mortality rates and resistant bacteria related infection rates in our ward during the study period. ${ }^{23-25}$ Mortality rates have been reported of different values with colistin monotherapy and combination therapy. Severity of illness and presence of comorbid risk factors, as well as timely and appropriate initiation of colistin, have been reported to influence patient survival. ${ }^{26-28}$ Colistin was recorded as monotherapy and combined therapy in our patients who died as well.

Although Enterococcal bacteremia is the third or fourth most common cause of nosocomial bacteremia depending on the region, and its rates are increasing worldwide, VRE bacteremia developed in only two of 50 (4\%) patients colonized with VRE. ${ }^{29} \mathrm{VRE}$ bacteremia is reported to be more closely related to the severity of the patient's condition than to the pathogenicity of the bacteria. ${ }^{30}$ The low frequencies of invasive procedures, including placement of chemotherapy port catheters and bone marrow biopsies, were likely to have reduced VRE-related BSI rates in the patients colonized with VRE. The VRE colonization rate was up to $40 \%$ among hematological cases under unfavorable conditions of our hematology ward. Unfavorable ward conditions, including 
shared toilets, housing attendants with patients, close contact between patients and attendants, frequent antibiotic use for infections, and immuno-suppression, were likely to have caused the high VRE colonization rates in our study. Those rates occurred in spite of infection control program for patients and their attendants as well as restricted use of glycopeptide and antianaerobic antibiotics in our hematology ward. Even if blood cultures are negative, episodes of VRE-related bacteremia should be considered in patients with neutropenia undergoing antimicrobial therapy and in those who present with persistent fever as well as worsening clinical signs and symptoms. Active VRE therapy should be initiated in such cases, because the mortality rates in patients colonized with VRE are 2.5 times higher than those of patients colonized with VSE. ${ }^{31}$

Prolonged and deep neutropenia, prolonged multiple antibiotic use, and unfavorable conditions were more likely to result in a higher incidence of IFIs in our cases. Colonization with yeast is commonly seen in hematology patients due to compromised immune status, use of broad-spectrum antibiotics, colonization of several body sites, disruption of physiological barriers in the digestive tract, and other factors. ${ }^{32}$ Hematology patients have a 12.5 times higher risk for candidemia related to use of multiple broad-spectrum antibiotics. ${ }^{33}$ AML, which poses a high risk for IFI, was diagnosed in 13 of 18 cases with fungal BSIs. Non-albicans Candida species caused most of the fungemia episodes in our cases. C. parapsilosis was isolated in one third of fungemia episodes in our patients. Whilst C. parapsilosis is commonly isolated from patients with vascular catheters and parenteral nutrition, C. tropicalis causes fungemia episodes in patients with cancer and neutropenia. ${ }^{34,35}$ C. krusei and C. glabrata fungemias generally occur in patients with previous exposure to azoles. ${ }^{35}$ A few studies from Turkey, USA, and Asia have reported that non-albicans species predominated among other species in candidemia episodes as $C$. parapsilosis predominated in our study, whereas other studies from Turkey and Europe revealed in favor of $C$. albicans. ${ }^{36-39}$ Whilst three of nine (33\%) patients whose catheters were removed died, five of nine $(55 \%)$ patients whose catheters were not removed survived. Although previous studies recommended removal of the catheter after identifying candidemia, Nucci et al reported that catheter removal by 24 or 48 hours after treatment initiation had no effect on overall treatment response, mortality, or mycological eradication rates in an analysis of 842 patients. ${ }^{40}$ Catheter removal should be evaluated with regard to the benefits and harms caused to the patient. Severity of illness and underlying conditions in patients are more likely to influence treatment response rates and the prognosis. In a study reported by Yildirmak et al, urinary catheterization, immunosuppressive therapy, APACHE II score ( $\geq 16)$, and hypoalbuminemia were described as independent risk factors for fatal candidemia. ${ }^{37}$ The overall incidence of candidemia in adults was calculated to be 4.19 episodes/10,000 admissions in the hospital where their study was performed. From 2006 to 2011 , incidence density rates were calculated as 2.38 $(n=5), 3.57(n=15), 5(n=21), 5.47(n=23), 4.04(n=17)$, and $3.8(n=16)$ per 10,000 admissions. ${ }^{37}$

IFIs that occur in hematology patients due to chemotherapy and immunosuppression lead to frequent and prolonged antifungal therapy, including azole antifungal drugs such as voriconazole, itraconazole, and fluconazole. Fungal pathogens resistant to voriconazole, fluconazole, amphotericin $\mathrm{B}$, and itraconazole were isolated in 10 of 19 cases who had exposure to azole antifungal drugs. Of the six cases whose antifungal drugs, including voriconazole $(n=2)$ and caspofungin $(n=1)$, have been switched to sensitive antifungal agents, three died due to voriconazole resistance $(\mathrm{n}=2)$ and caspofungin resistance $(n=1)$. Azole resistance has been emerging due to reported fluconazole resistance at $0.3 \%-10 \%$ and voriconazole-resistant Candida species that were isolated from patients who had a history of voriconazole therapy. ${ }^{37,42}$ Due to the fact that delayed or inappropriate antifungal treatment impairs survival rates, if a blood culture from a hematology patient yields yeast or fungi, antifungal treatment should be initiated taking into consideration local antifungal resistance status appropriately and rapidly. Since our three cases died even though antifungal therapy was switched to appropriate therapy. ${ }^{43}$

T. asahii and G. capitatum emerged as a cause of death in our patients with BSIs. Trichosporon was reported as the second most common cause of disseminated yeast infections and it can cause breakthrough fungal infection under CAS treatment as occurred in our study. ${ }^{44}$ Triazole (fluconazole, voriconazole) therapies are recommended with removing of central lines. ${ }^{45} \mathrm{G}$. capitatum caused two fungemia episodes that one case died under voriconazole treatment and other one case survived with voriconazole treatment and catheter removal achieving clinical and microbiological responses. G. capitatum (formerly called Trichosporon capitatum) is part of the normal flora of the human digestive and respiratory tracts and skin, and may become a pathogen in the event of disruption of the physiological barriers in the digestive tract in patients receiving chemotherapy. ${ }^{46}$ The catheter should be initially removed in the event of cultivation of $G$. capitatum in blood culture. Voriconazole and micafungin were reported to be successful treatment regimens. ${ }^{47}$ 
BSIs, which commonly occur during febrile neutropenic episodes by GNB in patients with hematological malignancies should be treated with non-carbapenem-based antipseudomonal antibiotic initially taking into consideration antimicrobial stewardship. Carbapenem resistance and then colistin based therapy should be considered in patient with risk factors for colonization or infection caused by carbapenem resistant bacteria. Non-azole antifungal drugs, including caspofungin and liposomal amphotericin B, should be preferred as empirical antifungal therapy in the event of possible or probable IFI with an absence of pulmonary findings due to increased azole resistance. However, T. asahii and G. capitatum should be kept in mind as emerging fungemia pathogens, taking into consideration voriconazole therapy and catheter removal.

\section{Disclosure}

The authors report no conflicts of interest in this work.

\section{References}

1. Crawford J, Dale DC, Lyman GH. Chemotherapy-induced neutropenia: risks, consequences, and new directions for its management. Cancer. 2004;100:228-237.

2. Carratala J, Gudiol F. Changing epidemiology of bacterial infection in neutropenic patients with cancer. Antibiot Chemother. 2000; $50: 1-9$.

3. Nørgaard M, Larsson H, Pedersen G, et al. Haematological malignancies - a predictor of a poor outcome in patients with bacteraemia. J Infect. 2006;53:190-198.

4. Pagano L, Tacconelli E, Tumbarello M, et al. Bacteremia in patients with haematological malignancies. Analysis of risk factors, etiological agents and prognostic indicators. Haematologica. 1997;82:415-419.

5. Nørgaard M. Risk of infections in adult patients with haematological malignancies. The Open Infectious Diseases Journal. 2012;6(Suppl 1 M4):46-51.

6. Sipsas NV, Lewis RE, Tarrand $\mathrm{J}$, et al. Candidemia in patients with haematologic malignancies in the era of new antifungal agents (2001-2007): stable incidence but changing epidemiology of a still frequently lethal infection. Cancer. 2009;115:4745-4752.

7. Marr KA, White TC, van Burik JA, Bowden RA. Development of fluconazole resistance in Candida albicans causing disseminated infection in a patient undergoing marrow transplantation. Clin Infect Dis. 1997; 25:908-910.

8. Freifeld AG, Bow EJ, Sepkowitz KA, et al. Clinical practice guideline for the use of antimicrobial agents in neutropenic patients with cancer: 2010 update by the Infectious Diseases Society of America. Clin Infect Dis. 2011;52:56-93.

9. Maertens J, Theunissen K, Verbeken E, et al. Prospective clinical evaluation of lower cut-offs for galactomannan detection in adult neutropenic cancer patients and haematological stem cell transplant recipients. $\mathrm{Br}$ J Haematol. 2004;126:852-860.

10. Caillot D, Couaillier JF, Bernard A, et al. Increasing volume and changing characteristics of invasive pulmonary aspergillosis on sequential thoracic computerised tomography scans in patients with neutropenia. J Clin Oncol. 2001;19:253-259.

11. Ascioglu S, Rex HB, De Pauw B, et al. Defining opportunistic invasive fungal infections in immunocompromised patients with cancer and hematopoietic stem cell transplants: an international consensus. Clin Infect Dis. 2002;34:7-14.
12. Braun E, Hussein K, Geffen Y, Rabino G, Bar-Lavie Y, Paul M. Predominance of Gram-negative bacilli among patients with catheterrelated bloodstream infections. Clin Microbiol Infect. January 24, 2014. [Epub ahead of print].

13. Bousquet A, Malfuson JV, Sanmartin N, et al. An 8-year survey strains identified in blood cultures in a clinical haematology unit. Clin Microbiol Infect. 2014;20:7-12.

14. Chen CY, Tsay W, Tang JL, et al. Epidemiology of bloodstream infections in patients with haematological malignancies with and without neutropenia. Epidemiol Infect. 2010;138:1044-1051.

15. Gedik H, Yıldırmak MT, Şimşek F, et al. The outcome of non-carbapenem-based empirical antibacterial therapy and VRE colonisation in patients with hematological malignancies. Afr Health Sci. 2013; 13: 362-368.

16. Blay JY, Chauvin F, Le Cesne A, et al. Early lymphopenia after cytotoxic chemotherapy as a risk factor for febrile neutropenia. J Clin Oncol. 1996;14:636-643.

17. Mendes RE, Farrell DJ, Sader HS, Jones RN. Comprehensive assessment of tigecycline activity tested against a worldwide collection of Acinetobacter spp. (2005-2009). Diagn Microbiol Infect Dis. 2010; 68:307-311.

18. Rosa RG, Goldani ZL, dos Santos RP. Risk factors for multidrug-resistant bacteremia in hospitalized cancer patients with febrile neutropenia: a cohort study. Am J Infect Control. 2014;42:74-76.

19. Huang ST, Chiang MC, Kuo SC, et al. Risk factors and clinical outcomes of patients with carbapenem-resistant Acinetobacter baumannii bacteremia. J Microbiol Immunol Infect. 2012;45:356-362.

20. Tumbarello M, Repetto E, Trecarichi EM, et al. Multidrug-resistant Pseudomonas aeruginosa bloodstream infections: risk factors and mortality. Epidemiol Infect. 2011;139:1740-1749.

21. Yang YS, Lee YT, Tsai WC, et al. Comparison between bacteremia caused by carbapenem resistant Acinetobacter baumannii and Acinetobacter nosocomialis. BMC Infect Dis. 2013;13:311.

22. Satlin MJ, Jenkins SG, Walsh TJ. The global challenge of carbapenemresistant enterobacteriaceae in transplant recipients and patients with hematologic malignancies. Clin Infect Dis. 2014;58:1274-1283.

23. Kochar S, Sheard T, Sharma R, et al. Success of an infection control program to reduce the spread of carbapenem-resistant Klebsiella pneumoniae. Infect Control Hosp Epidemiol. 2009;30:447-452.

24. Siegel JD, Rhinehart E, Jackson M, Chiarello L; Healthcare Infection Control Practices Advisory Committee. Management of multidrugresistant organisms in healthcare settings, 2006. Am J Infect Control. 2007;35(10 Suppl 2):165-193.

25. Centers for Disease Control and Prevention. 2012 CRE Toolkit: Guidance for control of carbapenem-resistant Enterobacteriaceae. Available from: http://www.cdc.gov./hai/organisms cre/cre-toolkit/index.html. Accessed April 5, 2014.

26. Lim SK, Lee SO, Choi SH, et al. The outcomes of using colistin for treating multidrug resistant acinetobacter species bloodstream infections. J Korean Med Sci. 2011;26:325-331.

27. Santimaleeworagun W, Wongpoowarak P, Chayakul P. Clinical outcomes of patients infected with carbapenem-resistant Acinetobacter baumannii treated with single or combination antibiotic therapy. J Med Assoc Thai. 2011;94:863-869.

28. Simsek F, Gedik H, Yildirmak MT, et al. Colistin against colistin-onlysusceptible Acinetobacter baumannii-related infections: monotherapy or combination therapy? Indian J Med Microbiol. 2012;30:448-452.

29. Mikulska M, Del Bono V, Prinapori R, et al. Risk factors for enterococcal bacteremia in allogeneic hematopoietic stem cell transplant recipients. Transpl Infect Dis. 2010;12:505-512.

30. Weinstock DM, Conlon M, Iovino C, et al. Colonization, bloodstream infection, and mortality caused by vancomycin-resistant enterococcus early after allogeneic hematopoietic stem cell transplant. Biol Blood Marrow Transplant. 2007;13:615-621.

31. Olivier CN, Blake RK, Steed LL, Salgado CD. Risk of vancomycinresistant Enterococcus (VRE) bloodstream infection among patients colonized with VRE. Infect Control Hosp Epidemiol. 2008;29:404-409. 
32. Vincent JL, Anaissie E, Bruining H, et al. Epidemiology, diagnosis and treatment of systemic Candida infection in surgical patients under intensive care. Intensive Care Med. 1998;24:206-216.

33. Wey SB, Mori M, Pfaller MA, Woolson RF, Wenzel RP. Risk factors for hospital-acquired candidemia. A matched case control study. Arch Intern Med. 1989;149:2349-2353.

34. Clark TA, Slavinski SA, Morgan J, et al. Epidemiologic and molecular characterization of an outbreak of Candida parapsilosis bloodstream infections in a community hospital. J Clin Microbiol. 2004;42: 4468-4472.

35. Bassetti M, Ansaldi F, Nicolini L, et al. Incidence of candidaemia and relationship with fluconazole use in an intensive care unit. J Antimicrob Chemother. 2009;64:625-629.

36. Messer SA, Jones RN, Fritsche TR. International surveillance of Candida spp. and Aspergillus spp.: report from the SENTRY Antimicrobial Surveillance Program (2003). J Clin Microbiol. 2006;44:1782-1787.

37. Yildirmak MT, Gedik H, Şimşek F, Iris NE, Gücüyener A. Is timely and appropriate antifungal drug enough for survival of adult cases with candidaemia? Five-year experience. West Indian Med J. 2013;62:724-730.

38. Yenigün BK, Kuloğlu F, Doğan ÇA, Akata F. [Evaluation of epidemiological characteristics and risk factors of candidemia in adult patients in a tertiary-care hospital]. Mikrobiyol Bul. 2011;45:489-503. Turkish.

39. Arendrup MC, Fuursted K, Gahrn-Hansen B, et al. Seminational surveillance of fungemia in Denmark: notably high rates of isolates with reduced azole susceptibility. J Clin Microbiol. 2005;43: 4434-4440.
40. Nucci M, Anaissie E, Betts RF, et al. Early removal of central venous catheter in patients with candidemia does not improve outcome: analysis of 842 patients from 2 randomized clinical trials. Clin Infect Dis. 2010;51:295-303.

41. Mermel LA, Allon M, Bouza E, et al. Clinical practice guidelines for the diagnosis and management of intravascular catheter-related infection: 2009 update by the Infectious Diseases Society of America. Clin Infect Dis. 2009;49:1-45.

42. Imhof A, Balajee SA, Fredricks DN, Englund JA, Marr KA. Breakthrough fungal infections in stem cell transplant recipients receiving voriconazole. Clin Infect Dis. 2004;39:743-746.

43. Morrell M, Fraser VJ, Kollef MH. Delaying the empiric treatment of candida bloodstream infection until positive blood culture results are obtained: a potential risk factor for hospital mortality. Antimicrob Agents Chemother. 2005;49:3640-3645.

44. Miceli MH, Diaz JA, Lee SA. Emerging opportunistic yeast infections. Lancet Infect Dis. 2011;11:142-151.

45. Colombo AL, Padovan AC, Chaves GM. Current knowledge of Trichosporon spp. and trichosporonosis. Clin Microbiol Rev. 2011;24: 682-700.

46. Martino P, Venditti M, Micozzi A, et al. Blastoschizomyces capitatus: an emerging cause of invasive fungal disease in leukemia patients. Clin Infect Dis. 1990;12:570-582.

47. Saghrounia F, Abdeljelila JB, Youssef YB, et al. Geotrichum capitatum septicemia in patients with acute myeloid leukemia. Report of three cases. Med Mycol Case Rep. 2012;1:88-90.
Therapeutics and Clinical Risk Management

\section{Publish your work in this journal}

Therapeutics and Clinical Risk Management is an international, peerreviewed journal of clinical therapeutics and risk management, focusing on concise rapid reporting of clinical studies in all therapeutic areas outcomes, safety, and programs for the effective, safe, and sustained use of medicines. This journal is indexed on PubMed Central, CAS,

\section{Dovepress}

EMBase, Scopus and the Elsevier Bibliographic databases. The manuscript management system is completely online and includes a very quick and fair peer-review system, which is all easy to use. Visit http://www.dovepress.com/testimonials.php to read real quotes from published authors. 